\title{
Resiliência de um cerradão submetido a perturbações intermediárias na transição Cerrado-Amazônia
}

\author{
Fernando Elias ${ }^{1}$ \\ Beatriz Schwantes Marimon 1,2* \\ Letícia Gomes ${ }^{1,2}$ \\ Mônica Forsthofer 1,2 \\ Mariângela Fernandes Abreu ${ }^{1}$ \\ Simone Almeida Reis ${ }^{1,2}$ \\ Eddie Lenza ${ }^{1,2}$ \\ Daniel David Franczak ${ }^{3}$ \\ Ben Hur Marimon-Junior 1,2

\footnotetext{
${ }^{1}$ Laboratório de Ecologia Vegetal, Departamento de Ciências Biológicas Universidade do Estado de Mato Grosso, Campus de Nova Xavantina Caixa Postal 08, CEP 78690-000, Nova Xavantina - MT, Brasil

${ }^{3}$ PPG em Botânica, Universidade de Brasília, Brasília - DF, Brasil

* Autor para correspondência

biamarimon@hotmail.com
} \\ ${ }^{2}$ PPG em Ecologia e Conservação, Universidade do Estado de Mato Grosso
}

Submetido em 28/08/2012

Aceito para publicação em 25/03/2013

\section{Resumo}

O objetivo do presente estudo foi testar a hipótese de que remanescentes de florestas, mesmo em pequenas unidades de conservação e sujeitos a perturbações intermediárias, são resilientes. Para isso, as distribuições de diâmetros e alturas da comunidade e das espécies mais abundantes de um cerradão (14² $42^{\prime} 02,3^{\prime \prime}$ S e $52^{\circ} 21^{\prime} 02,6^{\prime} \mathrm{W}$ ) foram analisadas e comparadas em intervalos de dois a três anos em um período de oito anos. Em 2002, 2005, 2008 e 2010 foram medidas todas as árvores vivas com diâmetro $\geq 5 \mathrm{~cm}$ a $0,3 \mathrm{~m}$ do solo em 50 parcelas de 10 x $10 \mathrm{~m}$. Mesmo com variações significativas na densidade, a distribuição de diâmetros e alturas da comunidade manteve o padrão de "J-reverso" e unimodal, respectivamente, indicando recrutamento contínuo e poucas mudanças na estrutura ao longo dos anos inventariados, corroborando a hipótese do presente estudo. Entre as espécies analisadas foram observados três padrões de distribuição diamétricas que provavelmente refletem diferentes estratégias de ocupação da floresta. Hirtella glandulosa foi a espécie mais apta a explorar os recursos do ambiente, apresentando as maiores abundâncias e indivíduos distribuídos em todas as classes de diâmetros.

Palavras-chave: Distribuição de diâmetros; Fogo; Mato Grosso; Seca; Unidades de conservação 


\section{Abstract}

Resilience of a cerradão subjected to intermediate disturbance in the Cerrado-Amazonia transition, Mato Grosso, Brazil. The present study examined the hypothesis that remnant forests in small protected areas may be resilient to intermediate disturbances. We analyzed the diameter and height distributions of the tree community in a typical cerradão ( $14^{\circ} 42^{\prime} 02.3^{\prime \prime S}$ and $\left.52^{\circ} 21^{\prime} 02.6^{\prime \prime} \mathrm{W}\right)$ and determined which species were most abundant, sampling at two- to three-year intervals over eight years. In 2002, 2005, 2008 and 2010, we measured all live trees with a diameter $\geq 5 \mathrm{~cm}$ at $0.3 \mathrm{~m}$ above ground in 50 plots of $10 \times 10 \mathrm{~m}$. Although significant variation was observed in the density of the trees and the distributions of their diameters and heights, the community maintained the "reverse-J" and unimodal patterns for these distributions, respectively. These results indicate continuous recruitment and little change in the structure of the community over the study period, supporting our hypothesis. Three different patterns of diametric distribution were observed among the analyzed species, likely reflecting different forest occupation strategies. Hirtella glandulosa was the speciesmost able to exploit its environment, as it possessed the greatest overall abundance and was represented by individuals in all diameter classes.

Key words: Conservation units; Diameter distribution; Drought; Fire; Mato Grosso

\section{Introdução}

O Brasil é líder mundial em desmatamento, registrando a conversão recorde de $19.500 \mathrm{~km}^{2}$ por ano de vegetação nativa em agricultura e pastagens entre 1996 e 2005 (NEPSTAD et al., 2009). Em Mato Grosso, as atividades agropecuárias estão ocupando imensas áreas, especialmente na faixa de contato entre o Cerrado e a Amazônia, região conhecida como o "arco do desmatamento" (FEARNSIDE, 2005; KLINK; MACHADO, 2005; NOGUEIRA et al., 2008), sendo que quase metade dos dois milhões de $\mathrm{km}^{2}$ originais do Cerrado já foram convertidos em atividades antrópicas (FEARNSIDE, 2006).

Por este motivo, Felfili e Silva-Júnior (1988) destacam a forte necessidade de estudos detalhados no Cerrado para subsidiar práticas sustentáveis com fins econômicos. Nesse caso, deve ser enfocada a aplicação de métodos de manejo adequado para as diversas fitofisionomias do bioma que promovam o desenvolvimento social e econômico nas áreas de fronteira agrícola sem riscos à conservação da biodiversidade (KLINK; MACHADO, 2005).

O manejo de ambientes florestais nativos requer uma detalhada e criteriosa compreensão dos complexos processos dinâmicos que envolvem tais áreas, sendo um grande desafio para os ecólogos (NAPPO et al., 2005). Neste contexto, a distribuição de diâmetros e alturas representada em histogramas de frequência, embora seja uma ferramenta simples, é bastante eficaz para o entendimento de importantes interações ecológicas, sendo utilizada para estudos de dinâmica, sucessão ecológica e conservação da biodiversidade (SILVA-JÚNIOR; SILVA, 1988; MARIMON; FELFILI, 2000; PAULA et al., 2004). Conhecendo a distribuição diamétrica de uma comunidade florestal e relacionando-a com eventos que podem interferir em seu desenvolvimento, como o fogo e a seca, é possível avaliar a sua resposta frente a estes distúrbios, gerando informações para o manejo com fins de conservação (FELFILI; SILVA-JÚNIOR, 1988).

Normalmente, as primeiras classes de diâmetro de florestas tropicais apresentam maior concentração de indivíduos, o que as caracterizam como estáveis, tendendo a uma diminuição à medida que aumenta o tamanho das classes diamétricas (SCOLFORO et al., 1998), caracterizando uma curva conhecida como "J-reverso", com um balanço equilibrado entre a mortalidade e o recrutamento dos indivíduos (população autorregenerativa). Daubenmire (1968) e Paula et al. (2004) observaram ainda que interrupções em uma ou mais classes de diâmetro indicariam condição de desequilíbrio.

O cerradão é uma fitofisionomia florestal que ocupava originalmente cerca de $1 \%$ da área total do bioma Cerrado, sendo composto de espécies comuns a outras fitofisionomias (RIBEIRO; WALTER, 2008; SOLÓRZANO et al., 2012). Além da reduzida área original, os cerradões se encontram severamente 
fragmentados, o que aumenta ainda mais o seu risco de instabilidade e extinção. Esta rara fitofisionomia ocorre nos extremos do gradiente ecotonal das formações savânicas do Cerrado, sendo considerada a mais ameaçada e fragilizada frente às perturbações no bioma (EITEN, 1979). Portanto, estudos que avaliem a estrutura destas áreas são essenciais, tendo em vista a escassez de informações sobre esta vegetação e a necessidade de sua preservação (MARIMON et al., 2006; SOLÓRZANO et al., 2012). Neste sentido, é importante ressaltar que não se trata de ameaça a uma espécie em particular, mas sim a toda uma fitofisionomia, a qual tem se tornado cada vez mais rara no Brasil.

Um estudo comparando diversas áreas de cerradão do Bioma Cerrado registrou que apesar desta fitofisionomia apresentar uma mistura de elementos florísticos savânicos e florestais, as áreas amostradas no estado de Mato Grosso apresentaram maior proporção de espécies florestais, evidenciando a influência da proximidade geográfica com a Amazônia (SOLÓRZANO et al., 2012). Ratter et al. (1973) sugerem a existência de dois tipos de cerradão que apresentam elevada riqueza de espécies na região leste de Mato Grosso, na zona de transição entre o Cerrado e a Amazônia, classificandoos de acordo com a pedologia e composição florística. O primeiro é o cerradão de Hirtella glandulosa, que geralmente ocorre em solos distróficos, e o segundo é o cerradão de Magonia pubescens e Callisthene fasciculata, que ocorre em solos mesotróficos, ambos apresentando elevada riqueza e diversidade florística. O Parque Municipal do Bacaba, em Nova Xavantina, MT, está situado na zona de transição entre os biomas Cerrado e Amazônia (MARIMON et al., 2006). No Parque ocorrem remanescentes de cerradão distrófico que apresentam diversidade florística superior àquelas encontradas em outros cerradões distróficos na região do Planalto Central (FRANCZAK et al., 2011). Estudos nestas áreas são urgentes, pois florestas transicionais, incluindo os cerradões no estado de Mato Grosso, estão sendo rapidamente convertidas em agricultura e pecuária pelo desmatamento (FEARNSIDE, 2006; MARIMON et al., 2006; NOGUEIRA et al., 2008).

O presente estudo teve por objetivo testar a hipótese de que remanescentes de florestas, mesmo em pequenas unidades de conservação e sujeitos a perturbações intermediárias, como fogo ocasional e eventos intensos de seca, apresentam resiliência, podendo manter sua estrutura por longo prazo. Para tanto, foram analisadas e comparadas as distribuições de diâmetros e alturas da comunidade e das principais espécies de um cerradão no Parque Municipal do Bacaba, Nova Xavantina, MT, no qual foram registrados, em um intervalo de oito anos (2002-2010), uma queimada e eventos intensos de seca.

\section{Material e Métodos}

\section{Área de estudo}

$\mathrm{O}$ estudo foi realizado em um remanescente de cerradão, com aproximadamente 15 ha, localizado a $14^{\circ} 42^{\prime} 02,3$ 'S e $52^{\circ} 21^{\prime} 02,6$ 'W, no Parque Municipal do Bacaba, unidade de conservação criada em 1995 (Lei Municipal no 52 de 27/12/1995), que ocupa aproximadamente 500 ha e está localizada na borda sudeste da transição entre os biomas Cerrado e Amazônia, no município de Nova Xavantina, leste de Mato Grosso (MARIMON-JUNIOR; HARIDASAN, 2005).

O clima da região é do tipo Aw, de acordo com a classificação de Köppen (KOTTEK et al., 2006), apresentando um período chuvoso (outubro a março) e um seco (abril a setembro), com precipitação anual em torno de $1.600 \mathrm{~mm}$ e temperatura média anual de $25^{\circ} \mathrm{C}$ (MARIMON et al., 2012). O relevo varia de plano a ondulado, com altitudes entre 250 e $300 \mathrm{~m}$, sendo o solo predominante do tipo Latossolo Amarelo álico distrófico (MARIMON-JUNIOR; HARIDASAN, 2005).

\section{Coleta de dados}

Foram demarcadas 50 parcelas de $10 \times 10 \mathrm{~m} \mathrm{e}$ medidas todas as árvores vivas com diâmetro $\geq 5 \mathrm{~cm}$ a $0,3 \mathrm{~m}$ do solo (DAS). Os indivíduos que apresentavam ramificações desde a base tiveram os ramos, desde que $\geq 5 \mathrm{~cm}$, medidos e os diâmetros determinados através da raiz quadrada da soma quadrática dos valores (SCOLFORO, 1993). Os diâmetros foram medidos com fita diamétrica e as alturas com régua telescópica graduada (indivíduos até $10 \mathrm{~m}$ ) ou trena a laser (maiores que $10 \mathrm{~m}$ ). 
Os inventários foram feitos sempre no mês de fevereiro, em 2002 (MARIMON-JUNIOR; HARIDASAN, 2005), em 2005 e 2008 (FRANCZAK et al., 2011) e em 2010 (presente estudo). Em setembro de 2008, depois que o inventário já havia sido realizado, a área de estudo foi atingida por uma queimada acidental que afetou parcialmente a vegetação (20 parcelas queimaram). Antes de 2008, há pelo menos 20 anos, não havia sido registrada nenhuma queimada na área de estudo.

No primeiro inventário, toda a vegetação lenhosa foi medida, identificada e marcada com plaquetas de alumínio numeradas para favorecer posteriores identificações. Nos inventários subsequentes todos os indivíduos sobreviventes foram remedidos e os recrutas (indivíduos que apresentaram limite mínimo de inclusão) foram medidos e plaqueteados. Amostras de todos os indivíduos foram coletadas, identificadas e comparadas com o acervo da coleção do Herbário NX (UNEMATNova Xavantina) e do Herbário UB (Universidade de Brasília), além de consultas a bibliografias e especialistas. Os espécimes férteis foram incorporados à coleção do Herbário NX e a classificação das famílias botânicas seguiu o sistema de classificação sugerido por APG III (2009).

\section{Análise de dados}

Para verificar se as densidades e os diâmetros médios e também as distribuições de diâmetros e alturas da comunidade e das principais espécies $(>20$ indivíduos em pelo menos três dos quatro inventários) diferiam entre os anos inventariados foi utilizado o teste de Kruskal-Wallis (KW) e, quando este indicou diferenças significativas, foi aplicado o teste de Dunn. Os testes de Shapiro-Wilk e Bartlett foram utilizados para testar a normalidade e homogeneidade dos dados, respectivamente (ZAR, 2010). Todas as análises foram feitas através do programa BioEstat 3.0, adotando-se o nível de significância de 5\% (AYRES et al., 2003). Para avaliar o balanço entre mortalidade e recrutamento em 2002, 2005, 2008 e 2010, para a comunidade e as principais espécies, foi utilizado o quociente ' $\mathrm{q}$ ' (LIOCOURT, 1898 apud SILVA-JÚNIOR; SILVA, 1988; FELFILI, 1997).
Os intervalos ideais de classes de altura e diâmetro foram determinados a partir da fórmula $\mathrm{IC}=\mathrm{A} / \mathrm{K}$, onde $\mathrm{A}$ representa a amplitude dos valores e $\mathrm{K}$ é uma constante definida pelo algoritmo de Sturges $\left(1+3,3 \times \log _{10} \mathrm{n}\right.$, sendo n o número total de indivíduos) (SPIEGEL, 1976). A primeira classe diamétrica foi iniciada com $4 \mathrm{~cm}$, pois alguns indivíduos amostrados em 2002, 2005 e 2008, e que apresentavam diâmetro mínimo de inclusão (DAS $\geq 5 \mathrm{~cm}$ ), perderam casca no inventário de 2010, após a passagem do fogo, apresentando assim DAS abaixo do limite mínimo aqui adotado.

\section{Resultados}

Distribuição de diâmetros e alturas da comunidade

Foram registrados na comunidade 942, 1.148, 1.136 e 813 indivíduos para os inventários de 2002, 2005, 2008 e 2010, respectivamente, com aumentos significativos nas densidades entre 2002 e 2008, antes da queimada, e redução significativa após o fogo, ou seja, em 2010 (Figura 1; Tabela 1). Os aumentos nas densidades de indivíduos, entre 2002 e 2008, ocorreram devido à entrada de recrutas na primeira classe diamétrica, principalmente entre os anos de 2002 e 2005 (Figura 1).

A distribuição dos diâmetros da comunidade nos quatro inventários apresentou o padrão de "J-reverso" (Figura 1), caracterizado pela concentração da maioria dos indivíduos nas classes de menores diâmetros, reduzindo exponencialmente para as classes maiores. Comparando a distribuição de diâmetros entre os anos inventariados foram verificadas diferenças significativas (KW, $\mathrm{H}=37,21, \mathrm{p}<0,0001$ ). Em 2005, foi registrado um aumento na densidade da maioria das classes diamétricas em relação a 2002. Entretanto, nos anos subsequentes, foi observada uma redução mais intensa nas menores classes (Figura 1). A média dos valores do quociente ' $q$ ' de Liocourt para as classes diamétricas estudadas apresentou aumento gradativo ao longo dos inventários, variando de 0,47 a 0,55 , com razão mais ou menos constante entre os inventários (Figura 1). 
FIGURA 1: Distribuição de diâmetros e valores do quociente ' $q$ ' de Liocourt no cerradão em 2002, 2005, 2008 e 2010, no Parque Municipal do Bacaba, Nova Xavantina, MT. Eventos de seca: 2005 e 2010 (sensu LEWIS et al., 2011); queimada: 2008, após o inventário. Total de indivíduos: 942 (2002), 1.148 (2005), 1.136 (2008) e 813 (2010).

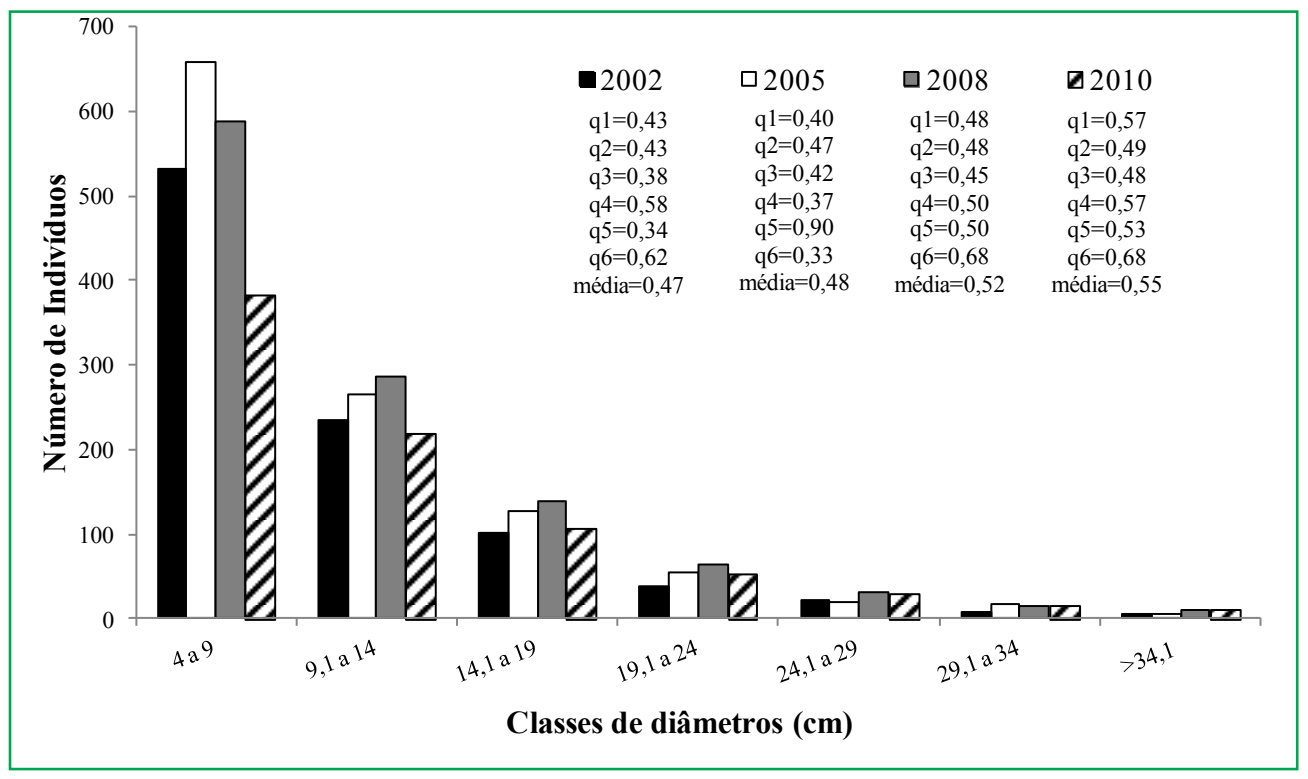

A distribuição de alturas do cerradão durante os períodos avaliados apresentou o padrão unimodal (Figura 2), com poucos indivíduos nas menores classes, muitos nas intermediárias e poucos nas classes maiores. Comparando a distribuição de alturas da comunidade
(Figura 2), foram verificadas variações significativas (KW, $\mathrm{H}=69,86, p=0,001$ ) entre os anos inventariados. A distribuição de alturas em 2002 apresentou diferenças em relação a todos os demais anos inventariados e a distribuição de 2005 diferiu apenas de 2010.

TABELA 1: Médias dos diâmetros e número de indivíduos por hectare (densidade) da comunidade e das populações das principais espécies no cerradão do Parque Municipal do Bacaba, Nova Xavantina, MT. As comparações entre os anos foram efetuadas através de estatística não-paramétrica. $(*)=p<0,05$ para os diâmetros; $(\#)=p<0,05$ para a densidade. Letras iguais representam valores estatisticamente similares usando o teste de Dunn. Espécies listadas em ordem decrescente do número de indivíduos no último inventário (2010).

\begin{tabular}{lrrrrrrrr}
\hline \multirow{2}{*}{\multicolumn{1}{c}{ Espécies e Famílias }} & \multicolumn{4}{c}{ Diâmetros (cm) } & \multicolumn{3}{c}{ Densidade (ind./ha) } \\
\cline { 2 - 8 } & $\mathbf{2 0 0 2}$ & $\mathbf{2 0 0 5}$ & $\mathbf{2 0 0 8}$ & $\mathbf{2 0 1 0}$ & $\mathbf{2 0 0 2}$ & $\mathbf{2 0 0 5}$ & $\mathbf{2 0 0 8}$ & $\mathbf{2 0 1 0}$ \\
\hline Hirtella glandulosa Spreng. (Chrysobalanaceae) & $14,0 \mathrm{a}$ & $15,7 \mathrm{a}$ & $16,7 \mathrm{a}$ & $16,6 \mathrm{a}$ & $222 \mathrm{a}$ & $242 \mathrm{a}$ & $254 \mathrm{a}$ & $242 \mathrm{a}$ \\
Tachigali vulgaris L.G. Silva \& H.C. Lima (Fabaceae) & $14,8 \mathrm{a}$ & $12,4 \mathrm{a}$ & $13,3 \mathrm{a}$ & $14,3 \mathrm{a}$ & $120 \mathrm{a}$ & $182 \mathrm{a}$ & $214 \mathrm{a}$ & $142 \mathrm{a}$ \\
Xylopia aromatica Mart. (Annonaceae)\# & $12,0 \mathrm{a}$ & $11,6 \mathrm{a}$ & $12,7 \mathrm{a}$ & $13,0 \mathrm{a}$ & $140 \mathrm{a}$ & $154 \mathrm{a}$ & $138 \mathrm{a}$ & $90 \mathrm{~b}$ \\
Tapirira guianensis Aubl. (Anacardiaceae) & $9,5 \mathrm{a}$ & $9,8 \mathrm{a}$ & $10,8 \mathrm{a}$ & $11,1 \mathrm{a}$ & $50 \mathrm{a}$ & $78 \mathrm{a}$ & $84 \mathrm{a}$ & $80 \mathrm{a}$ \\
Chaetocarpus echinocarpus (Baill.) Ducke (Peraceae)* & $7,4 \mathrm{a}$ & $8,1 \mathrm{ab}$ & $9,0 \mathrm{~b}$ & $9,2 \mathrm{~b}$ & $60 \mathrm{a}$ & $82 \mathrm{a}$ & $84 \mathrm{a}$ & $78 \mathrm{a}$ \\
Sorocea klotzschiana Baill. (Moraceae)* & $6,3 \mathrm{a}$ & $6,9 \mathrm{ab}$ & $7,8 \mathrm{~b}$ & $8,2 \mathrm{~b}$ & $26 \mathrm{a}$ & $56 \mathrm{a}$ & $62 \mathrm{a}$ & $60 \mathrm{a}$ \\
Myrcia splendens (Sw.) DC. (Myrtaceae) & $8,6 \mathrm{a}$ & $9,0 \mathrm{a}$ & $9,3 \mathrm{a}$ & $10,1 \mathrm{a}$ & $84 \mathrm{a}$ & $90 \mathrm{a}$ & $90 \mathrm{a}$ & $56 \mathrm{a}$ \\
Matayba guianensis Aubl. (Sapindaceae) & $8,9 \mathrm{a}$ & $9,0 \mathrm{a}$ & $9,5 \mathrm{a}$ & $9,8 \mathrm{a}$ & $62 \mathrm{a}$ & $74 \mathrm{a}$ & $74 \mathrm{a}$ & $50 \mathrm{a}$ \\
Aspidosperma multiflorum A. DC. (Apocynaceae) & $9,3 \mathrm{a}$ & $10,5 \mathrm{a}$ & $10,8 \mathrm{a}$ & $10,8 \mathrm{a}$ & $68 \mathrm{a}$ & $70 \mathrm{a}$ & $68 \mathrm{a}$ & $46 \mathrm{a}$ \\
Heisteria ovata Benth. (Olacaceae)* & $6,9 \mathrm{a}$ & $7,2 \mathrm{ab}$ & $7,7 \mathrm{ab}$ & $8,4 \mathrm{~b}$ & $52 \mathrm{a}$ & $88 \mathrm{a}$ & $82 \mathrm{a}$ & $46 \mathrm{a}$ \\
Siparuna guianensis Aubl. (Siparunaceae) & $7,3 \mathrm{a}$ & $7,4 \mathrm{a}$ & $7,7 \mathrm{a}$ & $7,6 \mathrm{a}$ & $12 \mathrm{a}$ & $38 \mathrm{a}$ & $42 \mathrm{a}$ & $44 \mathrm{a}$ \\
Vatairea macrocarpa (Benth.) Ducke (Fabaceae) & $10,0 \mathrm{a}$ & $9,7 \mathrm{a}$ & $9,9 \mathrm{a}$ & $11,8 \mathrm{a}$ & $46 \mathrm{a}$ & $52 \mathrm{a}$ & $56 \mathrm{a}$ & $34 \mathrm{a}$ \\
Comunidade *\# & $10,3 \mathrm{a}$ & $10,3 \mathrm{a}$ & $11,0 \mathrm{~b}$ & $11,6 \mathrm{~b}$ & $1.884 \mathrm{a}$ & $2.296 \mathrm{~b}$ & $2.272 \mathrm{~b}$ & $1.626 \mathrm{a}$ \\
\hline
\end{tabular}


FIGURA 2: Distribuição de alturas da comunidade do cerradão nos períodos de 2002, 2005, 2008 e 2010, no Parque Municipal do Bacaba, Nova Xavantina, MT. Eventos de seca: 2005 e 2010 (sensu LEWIS et al., 2011); queimada: 2008, após o inventário.

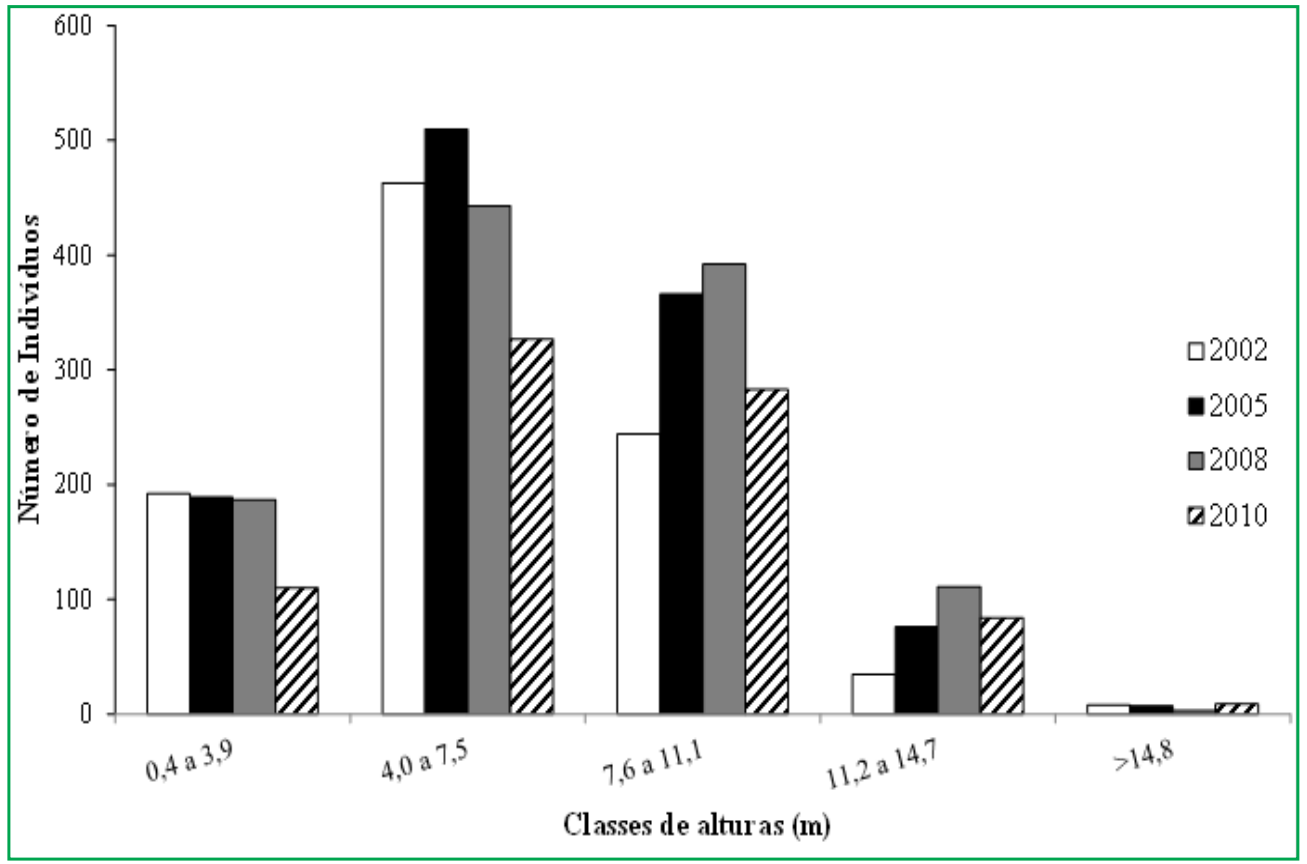

\section{Principais espécies}

Analisando a distribuição dos diâmetros das principais espécies na comunidade (Tabela 1), foram observados três padrões: o primeiro grupo foi representado por Tachigali vulgaris, Tapirira guianensis, Matayba guianensis e Vatairea macrocarpa (Figura 3). Neste caso, em todos os inventários o maior número de indivíduos encontra-se nas menores classes de diâmetro, sendo verificado um decréscimo gradual nas classes subsequentes. Foram registradas variações nas primeiras classes para Tachigali vulgaris e Tapirira guianensis, com a concentração de indivíduos nas classes de maiores diâmetros (ambas) e presença de classes interrompidas (apenas T. guianensis) (Figura 3). Indivíduos destas espécies constam entre os de maior altura na comunidade estudada, formando o dossel da floresta.

O segundo grupo foi representado por Hirtella glandulosa, Xylopia aromatica, Myrcia splendens e Aspidosperma multiflorum (Figura 4). Estas espécies, no primeiro inventário (2002), apresentaram um padrão similar ao registrado para as espécies do grupo anterior, com indivíduos concentrados nas menores classes e decréscimo gradual para as classes maiores.
Entretanto, de modo geral, foram observadas mudanças no padrão de distribuição das classes de diâmetros nos inventários dos anos subsequentes, destacando-se o fato de apresentarem menor número de indivíduos na primeira classe de diâmetros em relação à segunda (ex: Aspidosperma multiflorum em 2005, 2008 e 2010) ou alternância de picos e quedas em classes sucessivas (ex: Xylopia aromatica em 2008 e 2010) (Figura 4). Ocorreram variações na distribuição de diâmetros nas menores classes de $X$. aromatica, Myrcia splendens e A. multiflorum, especialmente no inventário de 2010 (Figura 4) e a densidade de $X$. aromatica apresentou diferenças significativas entre os inventários, com redução após o fogo de 2008 (Tabela 1).

No presente estudo, Hirtella glandulosa apresentou o maior diâmetro $(50,6 \mathrm{~cm})$, indivíduos em todas as classes (Figura 4) e também a maior densidade média (Tabela 1) em todos os levantamentos, sendo que em 2002 a população representou $11,7 \%$ da densidade total da comunidade, com um decréscimo para 10,5\% em 2005, seguido de aumentos para 11,1\% em 2008 e 14,8\% em 2010. 


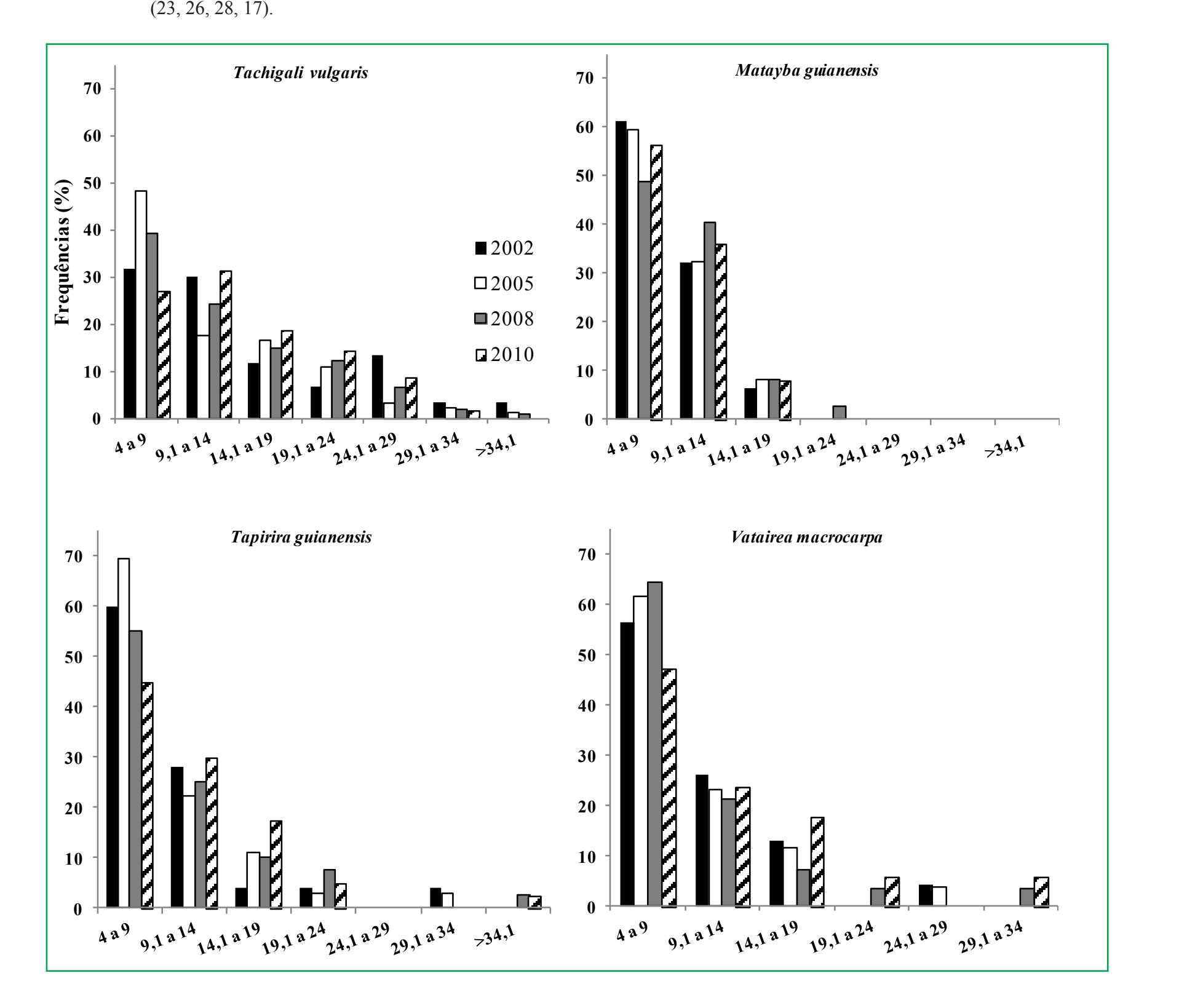


56

F. Elias et al.

FIGURA 4: Distribuição de diâmetros do segundo grupo das principais espécies do cerradão amostradas em 2002, 2005 , 2008 e 2010, no Parque Municipal do Bacaba, Nova Xavantina, MT. Número de indivíduos na área amostrada em 2002, 2005, 2008 e 2010, respectivamente: H. glandulosa $(111,121,127,121)$, X. aromatica $(70,77,69,45)$, M. splendens $(42,45,45,28)$ e A. multiflorum $(34,35,34,23)$.
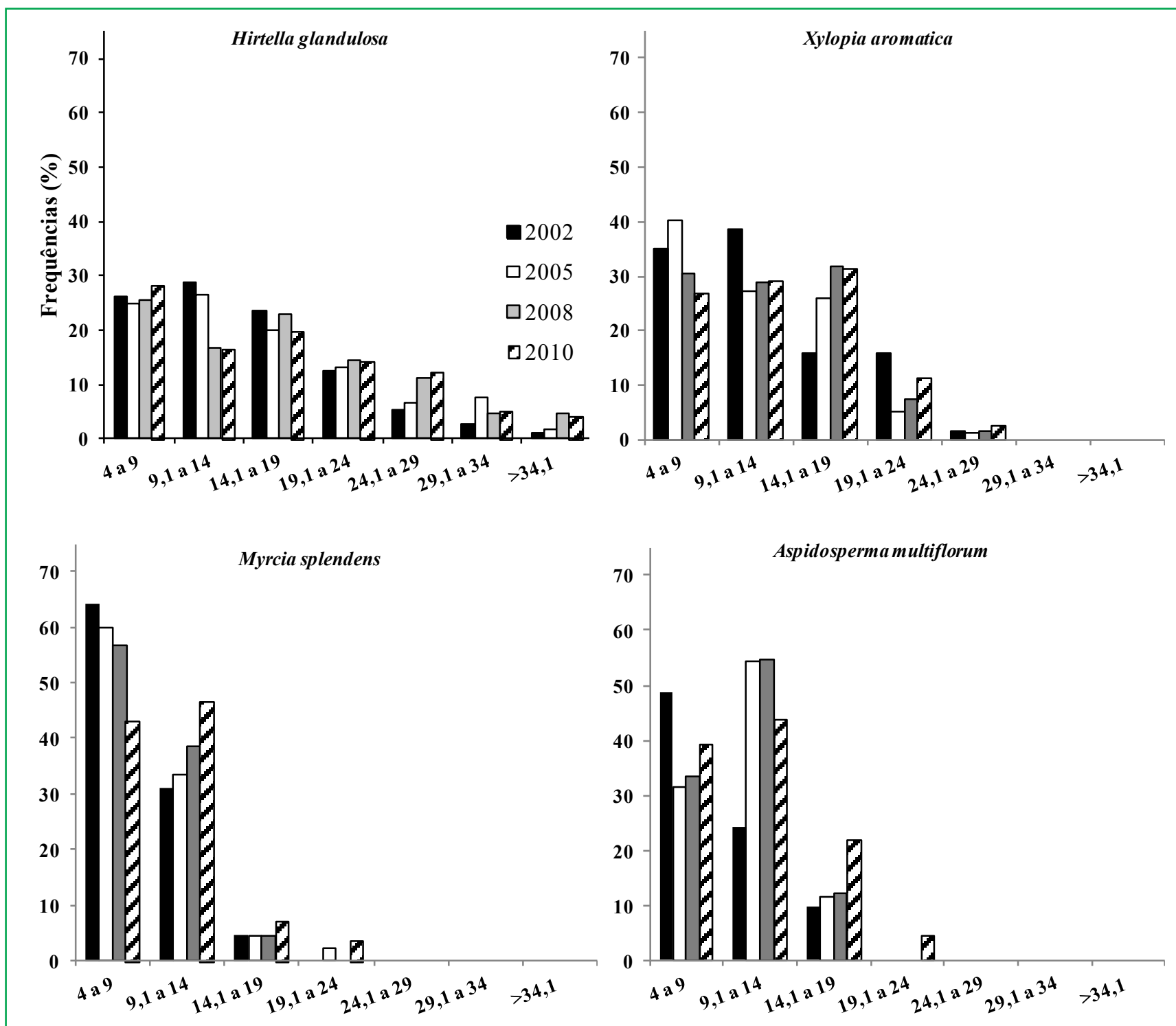

O terceiro grupo foi formado por Chaetocarpus echinocarpus, Sorocea klotzschiana, Heisteria ovata e Siparuna guianensis, com os indivíduos nos primeiros inventários (2002 e 2005) concentrados quase que integralmente na menor classe de diâmetro e caindo drasticamente nas classes seguintes (Figura 5). A média dos diâmetros entre os anos amostrados apresentou diferenças significativas para C. echinocarpus, $S$. klotzschiana e H. ovata, sempre com os maiores valores registrados no último inventário (Tabela 1).
No primeiro inventário (2002), cerca da metade dos indivíduos pertencia às 12 principais espécies e a outra metade às demais espécies (65). No decorrer dos anos, a proporção de indivíduos pertencentes às 12 principais espécies aumentou em relação às demais (Figura 6), confirmando uma predominância destas na comunidade.

Revista Biotemas, 26 (3), setembro de 2013 
Resiliência de um cerradão na transição Cerrado-Amazônia

57

FIGURA 5: Distribuição de diâmetros do terceiro grupo das principais espécies do cerradão amostradas em 2002, 2005, 2008 e 2010, no Parque Municipal do Bacaba, Nova Xavantina, MT. Número de indivíduos na área amostrada em 2002, 2005, 2008 e 2010, respectivamente: C. echinocarpus (30, 41, 42, 39), S. klotzschiana (13, 28, 31, 30), S. guianensis $(6,19,21,22)$ e H. ovata $(26,44,41,23)$.
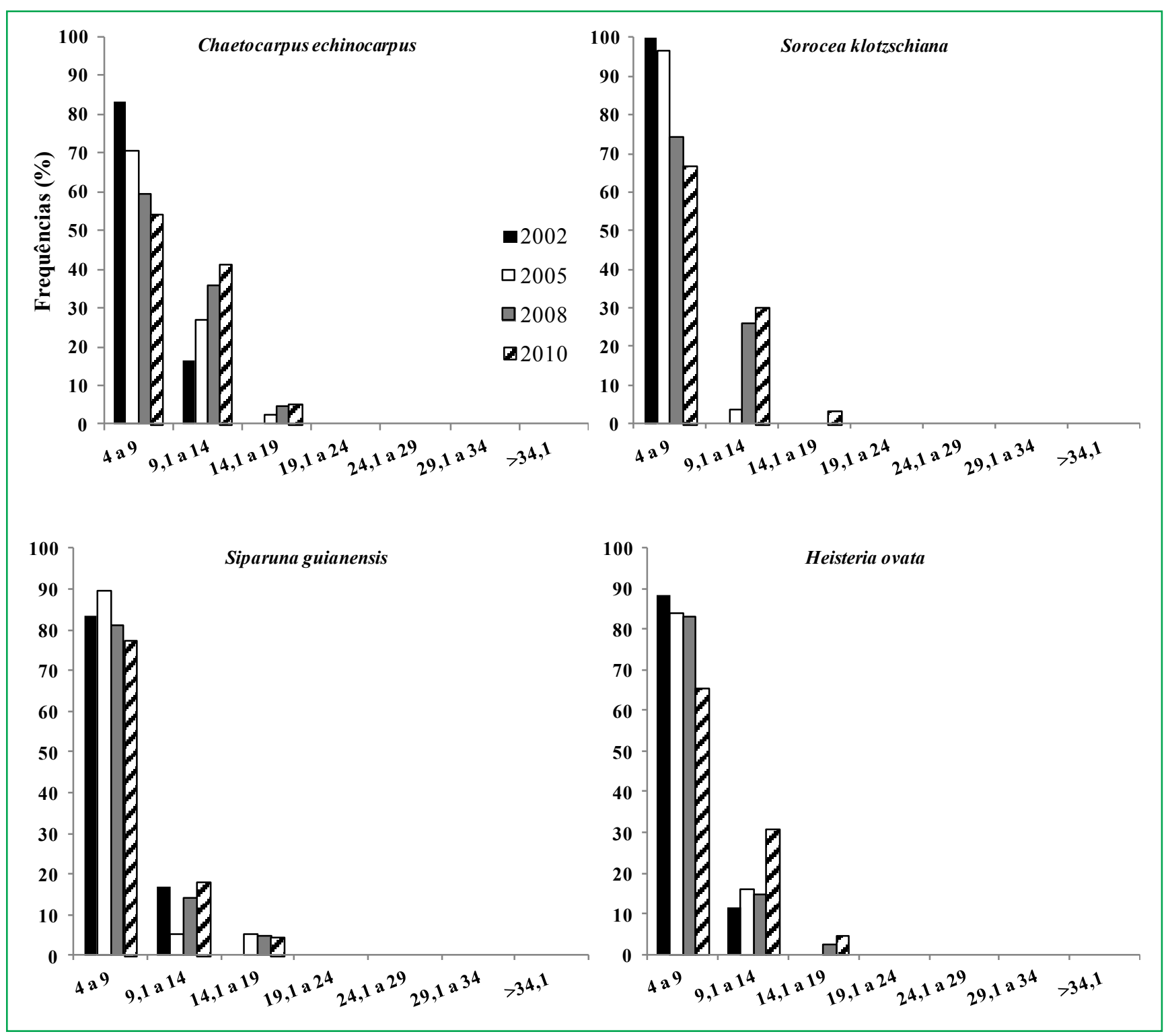

Revista Biotemas, 26 (3), setembro de 2013 
FIGURA 6: Proporção do número de indivíduos das 12 principais espécies $(\Delta)$ em relação às demais (匹) entre 2002, 2005, 2008 e 2010, no cerradão do Parque Municipal do Bacaba, Nova Xavantina, MT. Eventos de seca: 2005 e 2010 (sensu LEWIS et al., 2011); queimada: 2008, após o inventário.

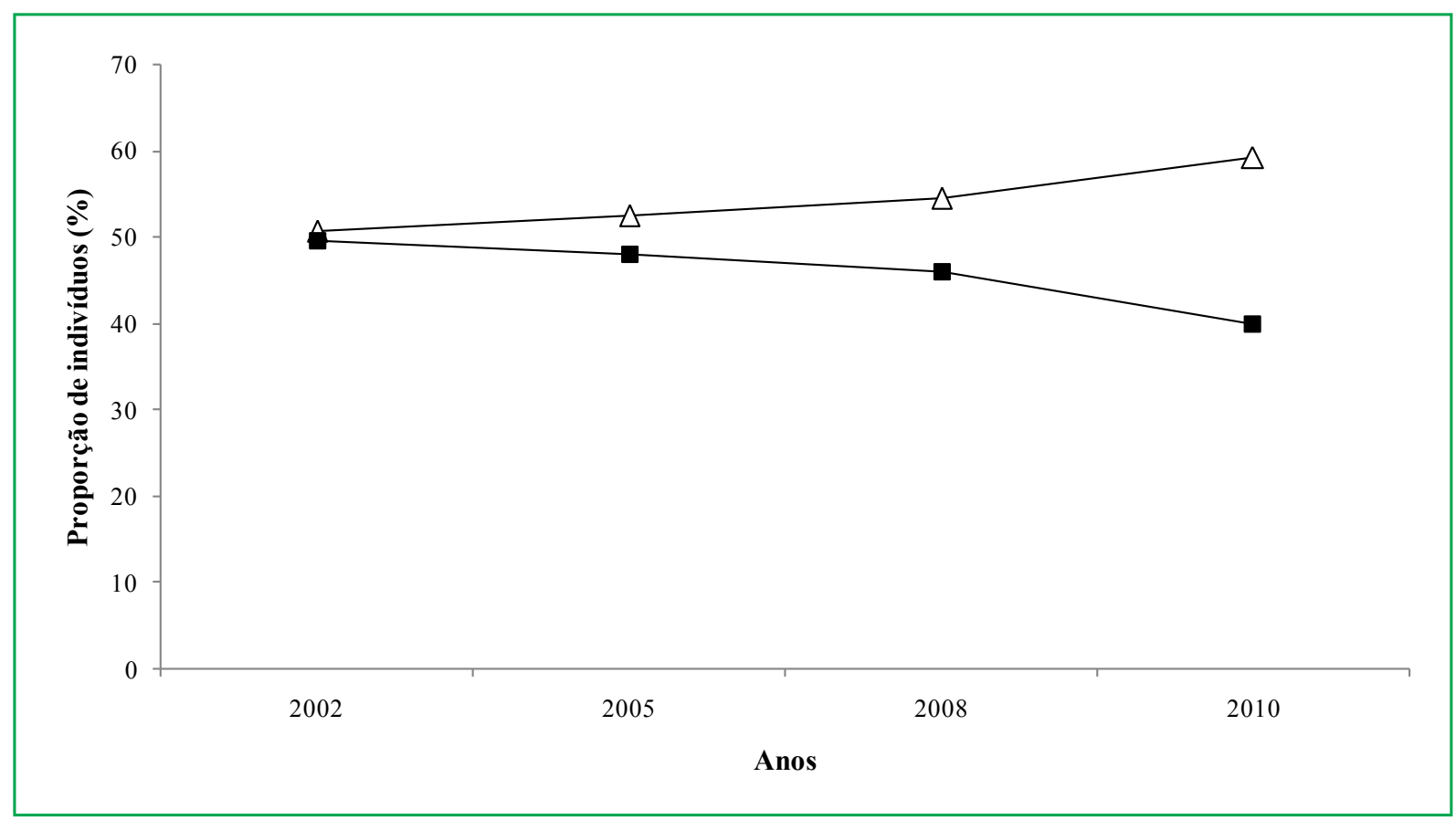

\section{Discussão}

Os aumentos registrados no número total de indivíduos da comunidade antes da queimada (942 a 1.136 ind., entre 2002 e 2008) e a redução após o fogo (813 ind., em 2010), bem como na densidade por hectare (vide Tabela 1), sugerem um impacto negativo deste no crescimento da comunidade. Lima et al. (2009), estudando o efeito do fogo na estrutura de uma área de cerrado sensu stricto em dois períodos, encontraram resultados semelhantes, onde a densidade reduziu consideravelmente após a passagem do fogo. Entretanto, apesar da densidade ter apresentando significativas diferenças entre os anos avaliados, o padrão de distribuição seguindo o modelo de "J-reverso" da comunidade (Figura 1) é típico de florestas que apresentam recrutamento contínuo ao longo dos anos (OLIVEIRA-FILHO et al., 1994; FELFILI, 1995, 1997; MEWS et al., 2012), confirmando que a mesma está conseguindo se manter. Esta situação é comumente encontrada em ambientes florestais preservados (FELFILI, 1997; CARVALHO; NASCIMENTO, 2009; MEWS et al., 2012), como é o caso do cerradão estudado, corroborando a hipótese do presente estudo, sugerindo que remanescentes de formações florestais podem se auto-manter em pequenas unidades de conservação, mesmo frente a perturbações intermediárias episódicas, como fogo. Entretanto, outros parâmetros importantes, como a composição e diversidade de espécies, também precisam ser considerados antes de se concluir que estes remanescentes são realmente resilientes às perturbações.

As variações observadas nas classes de diâmetros entre os inventários (Figura 1), como a elevada densidade na maioria das classes diamétricas em 2005, sugerem aumento nos níveis de estabelecimento e crescimento dos indivíduos. Marimon et al. (2012) observaram que na região onde foi realizado o presente estudo ocorreu um nível elevado de chuvas entre 2002 e 2004, quando foram registradas as maiores precipitações em um período de 15 anos. Neste caso, as chuvas podem ter contribuído para o crescimento em diâmetro e o aumento (migração) no número de indivíduos em cada classe diamétrica. Entretanto, as reduções na densidade das classes diamétricas nos anos subsequentes (Figura 1) indicam que a comunidade passou por perturbações que inibiram o crescimento dos indivíduos. 
Neste período, ocorreram expressivos eventos do fenômeno El Niño, que resultaram em secas severas na região, especialmente em 2005 (LEWIS et al., 2011). Desta forma, as variações no regime de chuvas, registradas por Lewis et al. (2011) e Marimon et al. (2012) durante a década de estudos, podem ter provocado parte das oscilações verificadas na dinâmica deste cerradão, mas não suficientes para comprometer a estabilidade da vegetação em longo prazo. Além disso, a área de estudo foi atingida pelo fogo em setembro de 2008, o que também pode ter causado a mortalidade e a redução de indivíduos nas referidas classes, pois segundo Felfili et al. (2000) e Barlow et al. (2003), as espécies podem desaparecer ou reduzir o número de indivíduos até três anos após a passagem do fogo, sendo que os mais afetados são os indivíduos jovens.

Apesar dos impactos (seca e fogo) não terem, aparentemente, comprometido a distribuição de diâmetros da comunidade no período estudado, foram registradas mudanças importantes na densidade da comunidade e de algumas espécies, o que poderá acarretar uma alteração funcional do cerradão como um todo. As variações que ocorreram entre as classes diamétricas apontadas pelo quociente ' $q$ ' de Liocourt foram mais ou menos constantes entre os inventários, porém as mudanças na densidade sugerem que a comunidade foi perturbada e o recrutamento nos próximos anos poderá ser afetado. Neste caso, tal como sugerido por Felfili e Silva-Júnior (1988), a estrutura do cerradão estudado pode ser definida como irregularequilibrada, com distribuição tendendo a balanceada e baixo comprometimento da estrutura da vegetação.

O padrão unimodal das alturas observado na comunidade (Figura 2) é normalmente encontrado em florestas tropicais que estão em processo de autoregeneração (FELFILI, 1995, 1997; MARIMON et al., 2001). A diferença observada na distribuição de alturas em 2002 com os demais anos (2005, 2008 e 2010) pode estar ligada ao fato de que no primeiro inventário a comunidade ainda não havia sofrido os efeitos da seca de 2005 e do fogo em 2008, conforme discutido para a distribuição de diâmetros. Moreira (2000), comparando um cerradão protegido do fogo por 18 anos e outro sujeito a queimadas com intervalos de dois anos, verificou que na área protegida as alturas foram superiores em quase todas as classes avaliadas confirmando que o fogo diminui consideravelmente a altura de algumas populações de espécies arbóreas.

A distribuição de diâmetros das 12 populações analisadas apresentou particularidades, provavelmente como reflexo das diferentes estratégias de cada população à dinâmica da floresta (OLIVEIRA-FILHO et al., 1994) e resposta particular aos distúrbios, sendo possível diferenciar três grupos com padrões similares de distribuição. A distribuição diamétrica do primeiro grupo indica, segundo Bongers et al. (1988), boa reprodução e um recrutamento contínuo ao longo dos anos. Os indivíduos pertencentes às espécies deste grupo estiveram entre os de maior altura na comunidade. Oliveira-Filho et al. (1994) observaram que espécies com este padrão de distribuição são tolerantes à sombra, apresentando bom recrutamento sob o dossel e habilidade para ocupar rapidamente os estratos superiores de uma floresta. A presença de classes interrompidas (Figura 3, Tabela 1), sugere que o ciclo de vida destas populações não está se completando (PAULA et al., 2004) e em algum momento o recrutamento não superou a mortalidade, indicando perturbação recente (MARIMON; FELFILI, 2000). Entretanto, o expressivo número de indivíduos nas menores classes reforça que estas populações apresentam potencial para se auto-regenerar e se estabelecer, caso não sofram perturbações intensas (FELFILI; SILVA-JÚNIOR, 1988; SILVA-JÚNIOR; SILVA, 1988).

Para o segundo grupo, Felfili e Silva-Júnior (1988) e Mews et al. (2012), destacam que espécies com este tipo de distribuição são compostas de populações maduras e desbalanceadas, onde o recrutamento não compensa a mortalidade, indicando problemas na regeneração natural ou perturbação recente, como foi o caso de Xylopia aromatica, Myrcia splendens e Aspidosperma multiflorum. As variações registradas na distribuição de diâmetros destas espécies, e na densidade ( $X$. aromatica), especialmente no inventário de 2010 , podem indicar sensibilidade ao fogo ou à seca, especialmente nas menores classes de diâmetro (Figura 4, Tabela 1). Outros fatores, como a capacidade de regeneração em ambientes 
sombreados, também precisam ser considerados para espécies que apresentam este padrão de distribuição de diâmetros.

A espécie observada como a mais apta para explorar os recursos do ambiente no cerradão foi Hirtella glandulosa, uma vez que foi registrada a presença de indivíduos em todas as classes de diâmetros e maior abundância em todos os levantamentos. Esta espécie ocorre com frequência em áreas de cerradão distróficos, como no presente estudo, e geralmente entre as espécies mais abundantes (RATTER et al., 1973; SOLÓRZANO et al., 2012). As oscilações observadas na densidade de H. glandulosa podem indicar que o recrutamento não superou a mortalidade em 2005. No entanto, o acréscimo expressivo nos anos subsequentes indica que a população está se auto-regenerando e se estabelecendo com êxito no cerradão, confirmando a resiliência desta fitofisionomia, mesmo na forma de um fragmento em uma pequena unidade de conservação.

As espécies que compõem o terceiro grupo (Figura 5), apesar de em casos excepcionais alcançarem alturas maiores, apresentam pequeno porte e são típicas do sub-bosque (MARIMON et al., 2001). De acordo com Oliveira-Filho et al. (1994), espécies que apresentam este padrão de distribuição de diâmetros são caracterizadas como tolerantes à sombra e podem ser abundantes onde um dossel mais aberto promove sombreamento moderado. $\mathrm{O}$ aumento sucessivo no diâmetro médio destas espécies (Tabela 1) indica ainda que estas estão crescendo na comunidade.

A tendência de dominância de algumas espécies tem sido descrita por vários autores em comunidades florestais (FELFILI, 1994; MARIMON et al., 2006; CARVALHO; NASCIMENTO, 2009) e o aumento da densidade de algumas populações no presente estudo, apesar dos distúrbios (seca e fogo) registrados na área, sugere que estas espécies estão bem adaptadas ao cerradão e se estabelecendo estruturalmente com êxito (SILVA-JÚNIOR; SILVA, 1988) (Figura 6) e, mesmo na presença de pequenos distúrbios, é possível que as mesmas se alterem pouco ao longo do tempo (FELFILI, 1994). Entretanto, algumas espécies apresentaram redução de densidade e suas populações poderão sofrer alterações drásticas no futuro. Neste caso, recomendamos que os efeitos do fogo na comunidade sejam melhor avaliados, tanto em termos de frequência quanto intensidade, pois apesar de apresentar resiliência não há nenhuma garantia de que esta comunidade se mantenha com o aumento da frequência das queimadas.

Ainda não sabemos se as alterações ocorreram em função dos distúrbios locais (fogo em 2008) ou de mudanças climáticas globais (seca em 2005 e 2010). Entretanto, é essencial avaliar esta tendência para que possamos compreender melhor as respostas das espécies e os novos padrões que passam a se configurar nas comunidades em uma escala temporal maior. Estudos que tragam mais informações sobre o efeito do fogo na dinâmica de florestas são indispensáveis, tendo em vista que este é um importante fator de mudanças no bioma Cerrado (RIBEIRO; WALTER, 2008). Neste caso, é necessário conhecer as mudanças na estrutura de diferentes fitofisionomias do Cerrado para subsidiar discussões e ações sobre as alterações/impactos no uso de áreas do bioma e o impasse "desenvolvimento" versus "conservação" (KLINK; MACHADO, 2005), principalmente na zona de transição entre o Cerrado e a Amazônia, onde fortes pressões de desenvolvimento causam anualmente impactos em grandes áreas e os níveis de desmatamento pelo fogo são alarmantes, incentivados principalmente pela agricultura e pecuária e aumentando substancialmente o chamado "arco do desmatamento" (FEARNSIDE, 2005; NOGUEIRA et al., 2008; NEPSTAD et al., 2009).

Neste contexto de cenários futuros imprecisos e de forte ameaça à sobrevivência das espécies, as pequenas unidades de conservação e os seus remanescentes florestais podem desempenhar um papel chave na manutenção da diversidade fitofisionômica do bioma Cerrado. Como muitas espécies não sobrevivem fora de seu habitat natural, a preservação das diferentes formações florestais da transição Cerrado-Amazônia, como os cerradões, é fundamental para a conservação da biodiversidade.

\section{Agradecimentos}

Ao Conselho Nacional de Desenvolvimento Científico e Tecnológico (CNPq/PELD - Projeto 
"Transição Cerrado-Floresta Amazônica: bases ecológicas e sócio-ambientais para a conservação", Processo no 558069/2009-6) pela concessão de bolsas e pelo financiamento deste estudo. Agradecemos a todos do Laboratório de Ecologia Vegetal que de alguma forma contribuíram com este trabalho.

\section{Referências}

APG III - ANGIOSPERM PHYLOGENY GROUP. An update of the angiosperm phylogeny group classification for the orders and families of flowering plants: APG III. Botanical Journal of the Linnean Society, London, v. 161, p. 105-121, 2009.

AYRES, M.; AYRES-JUNIOR, M.; AYRES, D. L.; SANTOS, A. S. BioEstat 3.0: aplicações estatísticas nas áreas das Ciências Biológicas e Médicas. Belém: Sociedade Civil Mamirauá/CNPq, 2003. 290 p.

BARLOW, J.; PERES, C. A.; LOGAN, B. O.; HAUGAASEN, T. Large tree mortality and the decline of forest biomass following Amazonian wildfires. Ecology Letters, London, v. 6, p. 6-8, 2003. BONGERS, F.; OPMA, J.; MEAVE DEL CASTILLO, J.; CARABIAS, J. Structure and floristic composition of the lowland rain forest of Los Tuxtlas, Mexico. Vegetatio, London, v. 74, p. 55-80, 1988.

CARVALHO, F. A.; NASCIMENTO, M. T. Estrutura diamétrica da comunidade das principais populações arbóreas de um remanescente de Floresta Atlântica Submontana (Silva Jardim RJ, Brasil). Revista Árvore, Viçosa, v. 33, n. 2, p. 327-337, 2009.

DAUBENMIRE, R. Plant communities: a textbook of plant synecology. New York: Harper \& Row Publ., 1968. 300 p.

EITEN, G. Formas fisionômicas do cerrado. Revista Brasileira de Botânica, São Paulo, v. 2, p. 139-148, 1979.

FEARNSIDE, P. Desmatamento na Amazônia: história, índices e consequências. Megadiversidade, Belo Horizonte, v. 1, n. 1, p. 113-123, 2005.

FEARNSIDE, P. Desmatamento na Amazônia: dinâmica, impactos e controle. Acta Amazonica, Manaus, v. 36, n. 3, p. 395-400, 2006.

FELFILI, J. M. Floristic composition and phytosociology of the gallery forest alongside the Gama stream in Brasília, DF, Brazil. Revista Brasileira de Botânica, São Paulo, v. 17, p. 1-11, 1994.

FELFILI, J. M. Diversity, structure and dynamics of a gallery Forest in central Brazil. Vegetatio, London, v. 117, p. 1-15, 1995.

FELFILI, J. M. Diameter and height distributions in a gallery forest tree community and some of its main species in central Brazil over a six-year period (1985-1991). Revista Brasileira de Botânica, São Paulo, v. 20, n. 2, p. 155-162, 1997.

FELFILI, J. M.; REZENDE, A. V.; SILVA-JÚNIOR, M. C.; SILVA, M. A. Changes in the floristic composition of cerrado sensu stricto in Brazil over a nine-year period. Journal of Tropical Ecology, Cambridge, v. 16, p. 579-590, 2000.

FELFILI, J. M.; SILVA-JÚNIOR, M. C. Distribuição dos diâmetros numa faixa de cerrado na Fazenda Água Limpa (FAL) em BrasíliaDF. Acta Botanica Brasilica, Feira de Santana, v. 2, n. 1/2, p. 85104, 1988.
FRANCZAK, D. D.; MARIMON, B. S.; MARIMON-JUNIOR, B. H.; MEWS, H. A.; MARACAHIPES, L.; OLIVEIRA, E. A. Changes in the structure of a savanna Forest over a six-year period in the Amazon-Cerrado transition, Mato Grosso state, Brazil. Rodriguésia, Rio de Janeiro, v. 62, n. 2, p. 425-436, 2011.

KLINK, C. A.; MACHADO, R. B. A conservação do Bioma Cerrado. Megadiversidade, Belo Horizonte, v. 1, n. 1, p. 147-155, 2005.

KOTTEK, M.; GRIESER, J.; BECK, C.; RUDOLF, B.; BUBEL, F. World Map of the Köppen-Geiger climate classification updated. Meteorologische Zeitschrift, Viena, v. 15, n.3, p. 259-263, 2006.

LEWIS, S. L.; BRANDO, P. M.; PHILLIPS, O. L.; VAN DER HEIJDEN, G. M. F.; NEPSTAD, D. The 2010 Amazon drought. Science, New York, v. 331, p. 554, 2011.

LIMA, E. S.; LIMA, H. S.; RATTER, J. A. Mudanças pós-fogo na estrutura e composição da vegetação lenhosa, em um cerrado mesotrófico, no período de cinco anos (1997-2002) em Nova Xavantina-MT. Revista Cerne, Lavras, v. 15, n. 4, p. 468-480, 2009.

MARIMON, B. S.; FELFILI, J. M. Distribuição de diâmetros e alturas na floresta monodominante de Brosimum rubescens Taub. na Reserva Indígena Areões, Água Boa-MT, Brasil. Revista Árvore, Viçosa, v. 24, n. 2, p. 143-150, 2000.

MARIMON, B. S.; FELFILI, J. M.; HARIDASAN, M. Studies in monodominant forests in eastern Mato Grosso Brazil: I. A forest of Brosimum rubescens Taub. Edinburgh Journal of Botany, Edinburgh, v. 58, n. 1, p. 123-137, 2001.

MARIMON-JUNIOR, B. H.; HARIDASAN, M. Comparação da vegetação arbórea e características edáficas de um cerradão e um cerrado sensu stricto em áreas adjacentes sobre solo distrófico no leste de Mato Grosso, Brasil. Acta Botanica Brasilica, Feira de Santana, v. 19, p. 915-928, 2005.

MARIMON, B. S.; LIMA, E. S.; DUARTE, T. G.; CHIEREGATTO, L. C.; RATTER, J. A. Observations on the vegetation of northeastern Mato Grosso, Brazil. IV. An analysis of the Cerrado-Amazonian Forest ecotone. Edinburgh Journal of Botany, Edinburgh, v. 63, n. 2/3, p. 323-341, 2006.

MARIMON, B. S.; FELFILI, J. M.; FAGG, C. W.; MARIMONJUNIOR, B. H.; UMETSU, R. K.; OLIVEIRA-SANTOS, C.; MORANDI, P. S.; LIMA, H. S.; NASCIMENTO, A. R. T. Monodominance in a forest of Brosimum rubescens Taub. (Moraceae): structure and dynamics of natural regeneration. Acta Oecologica, New York v. 43, p. 134-139, 2012.

MEWS, H. A.; MARIMON, B. S.; MARACAHIPES, L.; OLIVEIRA, E. A. Análise temporal das distribuições de diâmetros e alturas de uma Floresta Estacional Semidecidual na transição Cerrado-Floresta Amazônica, Leste do Mato Grosso, Brasil. Biotemas, Florianópolis, v. 25, n. 2, p. 33-43, 2012.

MOREIRA, A. G. Effects of fire protection on savanna structure in Central Brazil. Journal of Biogeography, London, v. 27, n. 4, p. 1021-1029, 2000.

NAPPO, M. E.; GRIFFTH, J. J.; MARTINS, S. V.; JÚNIOR, P. M.; SOUZA, A. L.; OLIVEIRA-FILHO, A. T. Dinâmica da estrutura diamétrica da regeneração natural de espécies arbóreas e arbustivas no sub-bosque de povoamento puro de Mimosa scabrella Bentham, em área minerada em Poços de Caldas, MG. Revista Árvore, Viçosa, v. 29, n. 1, p. 35-46, 2005. 
NEPSTAD, D. C.; SOARES-FILHO, B. S.; MERRY, F.; LIMA, A.; MOUTINHO, P.; CARTER, J.; BOWMAN, M.; CATTANEO, A.; RODRIGUES, H.; SCHWARTZMAN, S.; McGRATH, D. G.; STICKLER, C. M.; LUBOWSKI, R.; PIRIS-CABEZAS, P.; RIVERO, S.; ALENCAR, A.; ALMEIDA, O.; STELLA, O. The end of deforestations in the Brazilian Amazon. Science, New York, v. 326, p. 1350-1351, 2009.

NOGUEIRA, E. M.; NELSON, B. W.; FEARNSIDE, P. M.; FRANÇA, M. B.; OLIVEIRA, A. C. A. Tree height in Brazil's "arc of deforestation": shorter trees in south and southwest Amazonia imply lower biomass. Forest Ecology and Management, Amsterdan, v. 255, p. 2963-2972, 2008.

OLIVEIRA-FILHO, A. T.; SCOLFORO, J. R. S.; MELLO, J. M. Composição florística e estrutura comunitária de um remanescente de floresta semidecídua montana em Lavras, MG. Revista Brasileira de Botânica, São Paulo, v. 17, n. 2, p. 167-182, 1994.

PAULA, A.; SILVA, A. F.; JÚNIOR, P. M.; SANTOS, F. A. M.; SOUZA, A. L. Sucessão ecológica da vegetação arbórea em uma floresta estacional semidecidual, Viçosa, MG, Brasil. Acta Botanica Brasilica, Feira de Santana, v. 18, n. 3, p. 407-423, 2004. RATTER, J. A.; RICHARDS, P. N.; ARGENTE, G. E.; GIFFORD, D. R. G. Observations on the vegetation of northeastern Mato Grosso. Philosophical Transaction for the Royal Society of London, Series B; Biological Sciences, London, v. 226, n. 880, p. 449-492, 1973.
RIBEIRO, J. F.; WALTER, B. M. T. As principais fitofisionomias do bioma cerrado. In: SANO, S. M.; ALMEIDA, S. P; RIBEIRO, J. F. (Ed.). Cerrado: ecologia e flora. Brasília: Embrapa Informação Tecnológica, 2008. p. 153-212.

SCOLFORO, J. R. S. Mensuração florestal: crescimento florestal. Lavras: ESAL/FAEPE, Lavras, 1993. 138 p.

SCOLFORO, J. R. S.; PULZ, F. A.; MELO, J. M. Modelagem da produção, idade das florestas nativas, distribuição espacial das espécies e análise estrutural. In: SCOLFORO, J. R. S. (Org.) Manejo Florestal. Lavras: Editora UFLA/FAEPE, 1998. p. 189246.

SILVA-JÚNIOR, M. C.; SILVA, A. F. Distribuição dos diâmetros dos troncos das espécies mais importantes do cerrado na Estação Florestal de Experimentação de Paraopeba (EFLEX)-MG. Acta Botanica Brasilica, Feira de Santana, v. 2, n. 1/2, p. 107-126, 1988

SOLÓRZANO, A.; PINTO, J. R. R.; FELFILI, J. M.; HAY, J. D Perfil florístico e estrutural do componente lenhoso em seis áreas de cerradão ao longo do bioma Cerrado. Acta Botanica Brasilica, Feira de Santana, v. 26, n. 2, p. 328-341, 2012.

SPIEGEL, M. P. Estatística. São Paulo: Mc Graw-Hill, 1976. 580 p.

ZAR, J. H. Bioestatistical analysis. 5. ed. New Jersey: Prentice Hall, 2010. 944 p. 